\title{
Theory of temperature dependence of the Fermi surface-induced splitting of the alloy diffuse-scattering intensity peak
}

\author{
Igor Tsatskis, *** \\ Department of Earth Sciences, University of Cambridge, Downing Street, Cambridge CB2 3EQ, United Kingdom
}

(November 15, 2018)

\begin{abstract}
The explanation is presented for the temperature dependence of the fourfold intensity peak splitting found recently in diffuse scattering from the disordered $\mathrm{Cu}_{3} \mathrm{Au}$ alloy. The wavevector and temperature dependence of the self-energy is identified as the origin of the observed behaviour. Two approaches for the calculation of the self-energy, the high-temperature expansion and the $\alpha$ expansion, are proposed. Applied to the $\mathrm{Cu}_{3} \mathrm{Au}$ alloy, both methods predict the increase of the splitting with temperature, in agreement with the experimental results.
\end{abstract}

05.50+q, 64.60.Cn, 61.66.Dk, 71.18+y

Recently, in the first in situ experiment to resolve the fine structure of the equilibrium diffuse scattering intensity from the disordered $\mathrm{Cu}_{3} \mathrm{Au}$ alloy, Reichert, Moss and Liang [1] have observed a marked temperature dependence of the fourfold splitting of the (110) short-range order (SRO) diffuse intensity peak. The separation of the split maxima changed reversibly, increasing with temperature. The same behaviour of the splitting was also found in 22 by analysing results of the Monte Carlo (MC) simulations for the $\mathrm{Cu}_{0.856} \mathrm{Al}_{0.144}$ alloy [3]. The peak splitting (Fig. 1) is attributed to the indirect interaction of atoms via conduction electrons in an alloy whose Fermi surface has flat portions; the effective interatomic pair interaction itself has split minima in the reciprocal space, and their location is determined by the wavevector $2 \mathbf{k}_{F}$ spanning these flat portions of the Fermi surface [4]. As indicated in [1], current theoretical approaches fail to explain the observed behaviour. Indeed, the standard approximation for the SRO diffuse intensity, the KrivoglazClapp-Moss (KCM) formula [5], is

$$
I^{K C M}(\mathbf{k})=\frac{1}{1+2 c(1-c) \beta V(\mathbf{k})},
$$

where $I(\mathbf{k})$ is the intensity in Laue units, $c$ the concentration, $\beta=1 / T, T$ the temperature in energy units and $V(\mathbf{k})$ the Fourier transform of the combination $V_{i j}=$ $\left(V_{i j}^{A A}+V_{i j}^{B B}\right) / 2-V_{i j}^{A B}$ of potentials $V_{i j}^{\alpha \beta}$ with which an atom of type $\alpha$ at site $i$ interacts with an atom of type $\beta$ at site $j$. Eq. (11) predicts that positions of the $I(\mathbf{k})$ peaks coincide with those of the corresponding minima of $V(\mathbf{k})$; therefore, the splitting does not depend on $T$, if it is assumed that $V(\mathbf{k})$ is $T$-independent. This assumption is justified at least as far as positions of the $V(\mathbf{k})$ minima are concerned, since the $2 \mathbf{k}_{F}$ value should not change over the considered temperature range [1]. Besides, the MC calculations [3] in which the increase of the splitting with temperature was found [2] were carried out for the $T$-independent pair interaction parameters. On the other hand, the cluster variation method [6] which in most cases leads to a significant improvement of the results in comparison with the KCM approximation [7], is

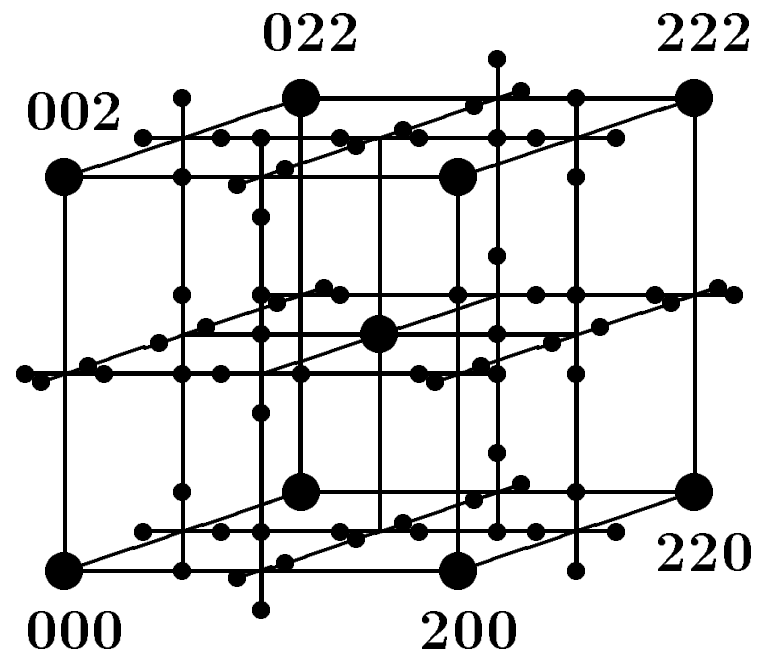

FIG. 1. Schematic reciprocal-space picture of scattering from the FCC alloys discussed in the text. Large circles represent the Bragg reflections, while small ones (forming characteristic crosses) correspond to the split diffuse intensity peaks.

practically inapplicable here, since interactions between atoms at distant lattice sites are involved (see below).

The aim of the present Letter is to propose the theory of the temperature-dependent peak splitting observed in the $\mathrm{Cu}_{3} \mathrm{Au}$ alloy. We begin by noting the exact expression for the SRO diffuse scattering intensity [B],

$$
I(\mathbf{k})=\frac{1}{c(1-c)[-\Sigma(\mathbf{k})+2 \beta V(\mathbf{k})]},
$$

where $\Sigma(\mathbf{k})$ is the so-called self-energy which depends not only on $\mathbf{k}$, but also on $c$ and $T$. In the KCM approximation, however, $\Sigma$ is $\mathbf{k}$ - and $T$-independent, as follows from the comparison of Eqs. (11) and (2):

$$
\Sigma^{K C M}=-[c(1-c)]^{-1} .
$$

Below we consider the $I(\mathbf{k})$ profile along one of the lines containing split peaks, e.g., the (h10) line, and concentrate on two peaks around the (110) position. The peak 


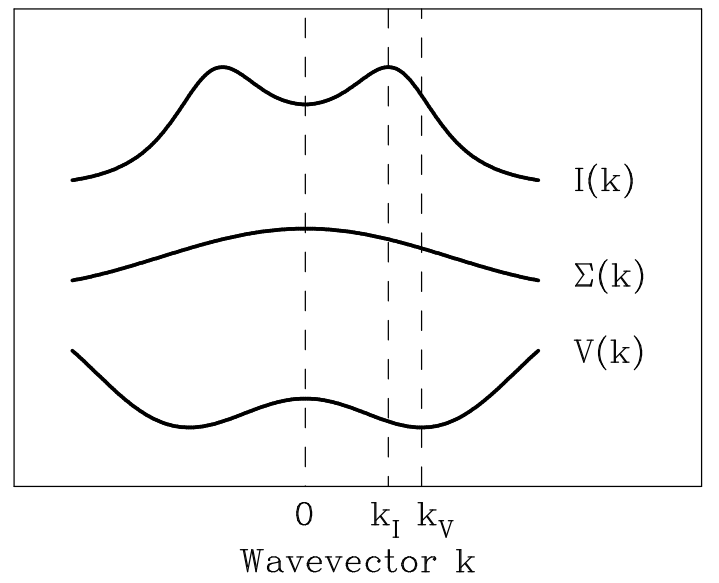

FIG. 2. Shift of the intensity peak position as a result of the wavevector dependence of the self-energy. The latter is as found for the $\mathrm{Cu}_{3} \mathrm{Au}$ alloy (see text).

positions $k_{I}$ ( $k$ is the deviation of the wavevector from the (110) position along the (h10) line) are determined by the condition $\partial_{k} I=0$ which gives

$$
2 \partial_{k} V=T \partial_{k} \Sigma .
$$

Eq. (1) means that the $\mathbf{k}$-dependence of $\Sigma$ leads to the shift $\delta k=k_{I}-k_{V}$ of the peak position with respect to the position $k_{V}$ of the corresponding minimum of $V(\mathbf{k})$ (Fig. 2); $k_{V}$ is the solution of the equation $\partial_{k} V=0$. Furthermore, the right side of Eq. (何) is a function of $T$, while its left side is $T$-independent. The $I(\mathbf{k})$ peaks will therefore change their positions with temperature.

At sufficiently high temperatures the behaviour of the splitting can be analysed by using the high-temperature expansion (HTE, in powers of $\beta V$ ) for $\Sigma$. The secondorder HTE approximation gives [9]

$$
\begin{aligned}
\left(\Sigma_{d}\right)_{i i} & =\Sigma^{K C M}-4 c(1-c) \beta^{2} \sum_{l} V_{i l}^{2}, \\
\left(\Sigma_{o d}\right)_{i j} & =2(1-2 c)^{2} \beta^{2} V_{i j}^{2} .
\end{aligned}
$$

Here $\Sigma_{d}$ is the diagonal and $\Sigma_{o d}$ the off-diagonal part of $\Sigma$. In this approximation Eq. (4) reduces to

$$
\partial_{k} V=(1-2 c)^{2} \beta \partial_{k} W, \quad W_{i j}=V_{i j}^{2} .
$$

The right side of Eq. (6) is small due to the prefactor $\beta$, and its solution $k_{I}$ deviates little from $k_{V}$. It is then sufficient to expand $\partial_{k} V$ and $\partial_{k} W$ in powers of the shift $\delta k$ and retain only linear terms,

$$
\begin{aligned}
\partial_{k} V(k) & =\left(\partial_{k}^{2} V\right)_{k_{V}} \delta k, \quad\left(\partial_{k}^{2} V\right)_{k_{V}}>0, \\
\partial_{k} W(k) & =\left(\partial_{k} W\right)_{k_{V}}+\left(\partial_{k}^{2} W\right)_{k_{V}} \delta k .
\end{aligned}
$$

Substituting Eqs. (7) into Eq. (6) and neglecting the last term in Eq. (7b $)$ because of the smallness of the right side of Eq. (6) lead to the result
TABLE I. Coefficients $B_{f}$ and $C_{f}$ (Eqs. (10)) for the interaction $V(k)$ and related function $W(k)$ as calculated from the inverse MC interactions $V_{l m n}$ 10 for the $\mathrm{Cu}_{3} \mathrm{Au}$ alloy at different temperatures.

\begin{tabular}{crcccc}
\hline \hline No. & $T, \mathrm{~K}$ & $B_{V}, \mathrm{meV}$ & $C_{V}, \mathrm{meV}$ & $B_{W},(\mathrm{meV})^{2}$ & $C_{W},(\mathrm{meV})^{2}$ \\
\hline 1 & 669 & -11.2 & 4.3 & 974.8 & 18.6 \\
2 & 678 & -11.9 & 2.8 & 202.6 & 8.1 \\
3 & 693 & -7.5 & 3.9 & 93.8 & 15.0 \\
4 & 723 & -3.8 & 0 & 131.6 & 0 \\
5 & 748 & -22.8 & 3.4 & 297.9 & 11.9 \\
6 & 958 & 6.7 & 0 & -13.7 & 0 \\
7 & 958 & -71.7 & 3.1 & 1657.1 & 9.6 \\
8 & 1023 & 17.7 & 2.1 & 285.7 & 4.3 \\
\hline \hline
\end{tabular}

$$
\delta k=(1-2 c)^{2}\left(\partial_{k} W\right)_{k_{V}} / T\left(\partial_{k}^{2} V\right)_{k_{V}} .
$$

Eq. (8) shows two scenarios for the temperature dependence of the splitting, depending on the sign of $\left(\partial_{k} W\right)_{k_{V}}$. The first is the increase of the splitting with temperature discussed above. Apart from that, the theory predicts that the decrease of the splitting with increasing temperature is also possible; such temperature dependence has not yet been observed. The absolute value of $\delta k$ decreases as $T^{-1}$ with temperature. The shifts of the two peaks have opposite signs and the same absolute values, and the wavevector dependence of $\Sigma$ determines whether the splitting increases or decreases with temperature. In the case of an equiatomic alloy $(c=0.5)$ the secondorder contribution to $\partial_{k} \Sigma$ is zero, and the temperature behaviour of the shift is defined by the higher-order terms in the HTE for the self-energy.

We now apply the HTE to the $\mathrm{Cu}_{3} \mathrm{Au}$ alloy for which sets of first 8 inverse $\mathrm{MC}$ interactions $V_{l m n}$ were obtained at different temperatures using SRO parameters $\alpha_{l m n}$ available in the literature [10]. Despite its extended range, the behaviour of the interaction along the (h10) line is simple; the Fourier transform $f(\mathbf{k})$ of an arbitrary FCC matrix $f_{i j}$ with non-zero elements for the first 20 coordination shells $l m n$ has along this line the form

$$
f(k)=A_{f}+2 B_{f} \cos 2 \pi k+2 C_{f} \cos 4 \pi k,
$$

where the relevant coefficients $B_{f}$ and $C_{f}$ are

$$
\begin{aligned}
B_{f}= & f_{200}-4 f_{211}+4 f_{220}+4 f_{222}-8 f_{321} \\
& +4 f_{420}-4 f_{332}+8 f_{422}-8 f_{521}+4 f_{442}, \\
C_{f}= & f_{400}-4 f_{411}+4 f_{420}+4 f_{422}-8 f_{431} \\
& +4 f_{440}-4 f_{433}+8 f_{442},
\end{aligned}
$$

and $k$ is measured in the reciprocal lattice units (r.l.u.). These coefficients for functions $V(k)$ and $W(k)$ are shown in Table I. All four quantities scatter widely, which is the result of relatively low accuracy of the inverse MC interactions for the $\mathrm{Cu}_{3} \mathrm{Au}$ alloy discussed in [10]. In particular, the accuracy and/or number of interactions are 
TABLE II. AE coefficients $B_{f}$ and $C_{f}$ (Eqs. (10)) for the self-energy $\Sigma(k)$ as calculated within the 10-shell approximation (except for set 16; see text) from the experimental sets of the SRO parameters for the $\mathrm{Cu}_{3} \mathrm{Au}$ alloy at different temperatures.

\begin{tabular}{|c|c|c|c|c|}
\hline No. & $T, \mathrm{~K}$ & $B_{\Sigma}$ & $C_{\Sigma}$ & Ref. \\
\hline 1 & 669 & 0.1742 & 0.0079 & 14 \\
\hline 2 & 693 & 0.0905 & 0.0119 & 14 \\
\hline 3 & 748 & 0.0915 & 0.0013 & 14 \\
\hline 4 & 958 & 0.0268 & 0.0022 & 14 \\
\hline 5 & 1023 & 0.0231 & 0.0002 & 14 \\
\hline 6 & 669 & 0.2172 & 0.0194 & 15 \\
\hline 7 & 693 & 0.0787 & 0.0141 & 15 \\
\hline 8 & 748 & 0.0592 & 0.0035 & 15 \\
\hline 9 & 958 & 0.0130 & 0.0042 & 15 \\
\hline 10 & 1023 & 0.0120 & 0.0006 & 15 \\
\hline 11 & 678 & 0.2086 & 0.0060 & 16 \\
\hline 12 & 733 & 0.1372 & 0.0004 & 16 \\
\hline 13 & 823 & 0.0530 & 0.0006 & 16 \\
\hline 14 & 678 & 0.4137 & 0.0144 & 17 \\
\hline 15 & 723 & 0.2053 & 0.0037 & 17 \\
\hline 16 & 678 & 0.1791 & 0 & 18 \\
\hline 17 & 703 & 0.0911 & 0.0021 & 19 \\
\hline
\end{tabular}

insufficient for the correct description of the split minimum of $V(k)$. The splitting in $V(k)$ occurs when $C_{V}>0$ and $\left|B_{V}\right|<4 C_{V}$, and this is so only for sets 1 and 3. In cases 4 and $6 C_{V}=0$, since the eighth interaction $V_{400}$ (the only one contributing to $C_{V}$ ) was not included in the corresponding sets. In addition, for cases 6 and 8 $B_{V}>0$, so that the split minimum would occur around the $\left(\frac{1}{2} 10\right)$ rather than (110) position. Nevertheless, despite low accuracy it is seen that $B_{W}$ is positive and $C_{W}$ is non-negative. The only exception is set 6 , where $B_{W}$ acquires very small negative value. However, the second set of interactions (set 7, with non-zero $V_{400}$ ) obtained using the same SRO parameters leads to positive values of both $B_{W}$ and $C_{W}$. In the case $B_{W}>0, C_{W} \geq 0$ function $W(k)$ has a maximum at the (110) position which is much wider than the magnitude of the peak splitting; $\left|k_{I}\right|$ values observed in [1] were quite small (less than 0.1 r.l.u.). As a result, the derivative $\partial_{k} W$ is positive for the left and negative for the right minimum of $V(k)$ (Fig. 2), and at any finite temperature the intensity peaks are shifted towards the (110) position. The absolute value of the shift increases with decreasing temperature, so that the splitting increases with temperature.

The applicability of the HTE, similarly to that of the KCM approximation, is limited to the case of sufficiently high temperatures. To deal with moderate temperatures, we introduce here another approach which leads to the $\mathbf{k}$ and $T$-dependence of $\Sigma$, its expansion in powers of SRO parameters $\alpha_{i j}$ (hereafter the $\alpha$-expansion, or AE). Two non-zero orders of the AE for $\Sigma_{o d}$ were calculated 8 in the framework of the $\gamma$-expansion method (GEM) 8, 11:

$$
\begin{aligned}
\left(\Sigma_{o d}\right)_{i j} & =a \alpha_{i j}^{2}+b \alpha_{i j}^{3}+O\left(\alpha^{4}\right), \\
a & =\frac{(1-2 c)^{2}}{2[c(1-c)]^{2}}, \\
b & =\frac{[1-6 c(1-c)]^{2}-3(1-2 c)^{4}}{6[c(1-c)]^{3}} .
\end{aligned}
$$

The expression for $\Sigma_{d}$ then comes from the sum rule

$$
\alpha_{i i}=\Omega^{-1} \int d \mathbf{k} I(\mathbf{k})=1
$$

(here the integration is carried out over the Brillouin zone of volume $\Omega$ ), Eq. (12) being one of the AE (or GEM) equations:

$$
\begin{aligned}
\left(\Sigma_{d}\right)_{i i}= & \Sigma^{K C M}+2 \beta \sum_{j} V_{i j} \alpha_{i j} \\
& -\sum_{j(\neq i)}\left(a \alpha_{i j}^{3}+b \alpha_{i j}^{4}\right)+O\left(\alpha^{5}\right) .
\end{aligned}
$$

Note that the sum of the first two terms corresponds to the spherical model (SM) for SRO [12], which is the zeroorder approximation for the AE and GEM; in the SM the self-energy is diagonal $(a=b=0)$. The difference between the AE and GEM lies in the choice of the expansion parameter(s). The GEM parameter is $\gamma=\exp (-1 / \xi), \xi$ being the dimensionless correlation length, and terms in the diagrammatic expansion for the self-energy are selected according to the total length of all lines in the diagrams, where the line connecting sites $i$ and $j$ represents $\alpha_{i j}$. The GEM is based on the assumption that the correlations decrease rapidly with distance; this assumption is invalid here because distant interactions are essential. The AE uses $\alpha_{i j}$ themselves as the expansion parameters; the terms are chosen according to the number of lines in the diagrams (i.e., the powers of $\alpha_{i j}$ ), since all $\alpha_{i j}$ are sufficiently small. The GEM was successfully applied to both the direct and inverse problems of alloy diffuse scattering [8,13], leading to reliable results everywhere except in the vicinity of the instability point. Based on our experience with GEM, we can as well expect the AE to be quite accurate at almost all temperatures.

Applying the $\mathrm{AE}$ to the $\mathrm{Cu}_{3} \mathrm{Au}$ alloy, we calculate coefficients $B_{\Sigma}$ and $C_{\Sigma}$ combining Eqs. (10) and (11) and using available sets of experimental SRO parameters 14 19]. Their values for the case of first 10 shells included in the AE approximation (11) for the self-energy are given in Table II (5-shell AE approximation was used for set 16 , since only 5 SRO parameters were determined in [18]). Inclusion of additional shells does not alter the results. In all cases $B_{\Sigma}$ is positive and $C_{\Sigma}$ is non-negative, so that, as before, $\Sigma(k)$ has a maximum at the (110) position which is very wide in comparison with the peak splitting, and the intensity peaks are shifted towards this position. Contrary to the case of the HTE, the explicit temperature dependence of the AE self-energy is unknown, 


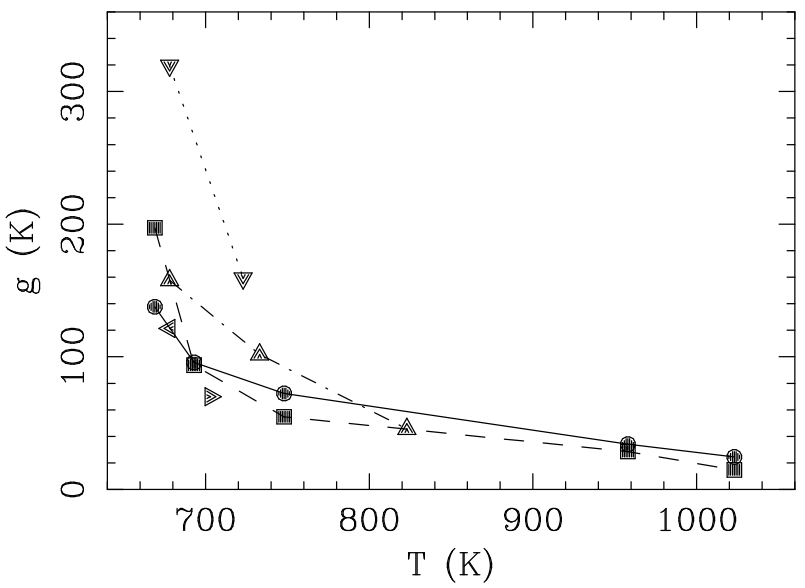

FIG. 3. Values of $g$ (Eq. (14)) vs. temperature calculated using the data from Table II [14] - dots, solid line; [15] squares, dashed line; 16 - upward triangles, dot-dashed line; [17] - downward triangles, dotted line; 18 - leftward triangle; [19] - rightward triangle.

since the SRO parameters in Eqs. (11) are complicated functions of temperature. To find the temperature behaviour of the splitting, we use the data from Table [1 and plot in Fig. 3 against temperature a quantity

$$
g=T\left|\partial_{k}^{2} \Sigma\right|_{k=0} / 8 \pi^{2}=T\left(B_{\Sigma}+4 C_{\Sigma}\right),
$$

which characterises the temperature dependence of the right side of Eq. (何) at small $k$. It is seen that $g$ is a decreasing function of $T$, which corresponds to the increase of the splitting with temperature. Its temperature dependence is particularly strong in the range below $800 \mathrm{~K}$, where the intensity profile was measured in [1].

To summarize, we have presented the explanation of the temperature dependence of the Fermi surface-induced diffuse intensity peak splitting found recently for the $\mathrm{Cu}_{3} \mathrm{Au}$ alloy. The wavevector and temperature dependence of the self-energy is understood to be the origin of this behaviour. The proposed theory is able to describe the observed increase of the peak separation with temperature; in addition, it also predicts the possibility for the splitting to decrease as temperature increases, the behaviour which has not yet been found. Two methods for the calculation of the $\mathbf{k}$ - and $T$-dependent self-energy, the HTE and the AE, have been proposed. Applied to the existing experimental and inverse $\mathrm{MC}$ data for the $\mathrm{Cu}_{3} \mathrm{Au}$ alloy, both methods predict the increase of the splitting with temperature, in agreement with the experimental findings. However, the HTE is not expected to be reliable when applied to alloys at realistic temperatures, so that the AE approach is preferable. Despite the seeming complexity of the problem (the interaction involves many coordination shells), the theoretical analysis proves to be surprisingly simple.

The author is grateful to S.C. Moss and H. Reichert for communicating their experimental results prior to publication and stimulating discussions.

* Electronic address: it10001@cus.cam.ac.uk

** Former name: I. V. Masanskii

[1] H. Reichert, S.C. Moss and K.S. Liang, Phys. Rev. Lett. 77, 4382 (1996).

[2] S.C. Moss and H. Reichert (private communication); H. Reichert, I. Tsatskis and S.C. Moss, in Proceedings of the Joint NSF/CNRS Workshop on Alloy Theory, Mont Sainte Odile Monastery, Strasbourg, France, October 1115, 1996, Comput. Mater. Sci. 8, 46 (1997).

[3] H. Roelofs et al., Scripta Mat. 34, 1393 (1996).

[4] M.A. Krivoglaz and Hao Tyu, Defects and Properties of the Crystal Lattice (Naukova Dumka, Kiev, 1968); S.C. Moss, Phys. Rev. Lett. 22, 1108 (1969); S.C. Moss and R.H. Walker, J. Appl. Crystallogr. 8, 96 (1974); M.A. Krivoglaz, Diffuse Scattering of X-Rays and Neutrons by Fluctuations (Springer, Berlin, 1996).

[5] M.A. Krivoglaz, Theory of X-Ray and Thermal Neutron Scattering by Real Crystals (Plenum, New York, 1969); P.C. Clapp and S.C. Moss, Phys. Rev. 142, 418 (1966); 171, 754 (1968).

[6] R. Kikuchi, Phys. Rev. 81, 988 (1951).

[7] E.g., D. de Fontaine, Solid State Physics 34, 73 (1979); 47, 33 (1994); F. Ducastelle, Order and Phase Stability in Alloys (North-Holland, Amsterdam, 1991).

[8] V.I. Tokar, I.V. Masanskii, and T.A. Grishchenko, J. Phys. Condens. Matter 2, 10199 (1990); I.V. Masanskii, V.I. Tokar, and T.A. Grishchenko, Phys. Rev. B 44, 4647 (1991).

[9] I. Tsatskis, in Local Structure from Diffraction, Fundamental Materials Science Series, eds. M.F. Thorpe and S.J.L. Billinge (Plenum Press, New York, 1998, in press).

[10] V. Gerold and J. Kern, Acta Metall. 35, 393 (1987).

[11] V.I. Tokar, Phys. Lett. A 110, 453 (1985); T.A. Grishchenko, I.V. Masanskii, and V.I. Tokar, J. Phys. Condens. Matter 2, 4769 (1990); I.V. Masanskii and V.I. Tokar, J. Phys. I France, 2, 1559 (1992).

[12] E.g., D.W. Hoffman, Metall. Trans. 3, 3231 (1972).

[13] L. Reinhard and S.C. Moss, Ultramicr. 52, 223 (1993); M. Borici-Kuqo and R. Monnier, Ref. [⿰]月, p. 16; D. Le Bolloc'h et al., Ref. [2], p. 24.

[14] H. Chen, R.J. Comstock and J.B. Cohen, Ann. Rev. Mater. Sci. 9, 51 (1979).

[15] P. Bardhan and J.B. Cohen, Acta Crystallogr. A 32, 597 (1976).

[16] J.M. Cowley, Phys. Rev. 77, 669 (1950).

[17] S.C. Moss, J. Appl. Phys. 35, 3547 (1964).

[18] C.B. Walker and D.T. Keating, J. Appl. Phys. 34, 2309 (1963).

[19] B.D. Butler and J.B. Cohen, J. Appl. Phys. 65, 2214 (1989). 